\title{
Enhancement of New Channel Equalizer Using Adaptive Neuro Fuzzy Inference System
}

\author{
P.Sivakumar ${ }^{1}$ L.Arthi ${ }^{2}$ \\ Asst Professor ${ }^{l}$ PG Scholar ${ }^{2}$ SKP Engineering college ${ }^{1,2}$, Thiruvannamalai.
}

\begin{abstract}
The rapidly increasing need for information communication requires higher speed data transmission over the existing channels. The data rate over these channels is limited mainly by Inter Symbol Interference (ISI). Channel equalizers are used to reduce the effect of ISI. In this paper, a new equalizer based on Adaptive Neuro-Fuzzy Inference System is presented. The performance of the proposed equalizer is evaluated for both linear as well as non-linear channels in terms of bit-error rate for different noise powers. Simulation results show that the proposed equalizer has better Bit Error Rate (BER) performance compared to multi-layer perceptron and least mean square equalizers. However, its BER performance is slightly poorer than that of radial basis function network and optimal Bayesian equalizer but is better in terms of structural complexity.
\end{abstract}

Keywords: Channel equalizer, Hybrid learning algorithm, Intersymbol interference, Membership function, optimal Bayesian equalizer.

\section{Introduction}

Voice bandwidth channels developed for voice communication have been used to transmit higher speed data to fulfill the increasing demand of computer and mobile communication networks. Frequency response of voice channels such as telephone lines deviates from the ideal low pass filter response, which cause time dispersion resulting in ISI. ISI is a major problem for reliable and efficient high speed data transmission over such bandwidth limited channels. Equalizers are used to compensate for the ISI and provide reliable data transmission over high speed communication channels that are impaired by the ISI . In most of the practical situations, the channel response is not known a priori and varies with time. In such cases, adaptive equalizers are designed so that they automatically adapt to the unknown channels and track the variations in the channel continuously

The problem of equalization is treated in two different ways. Firstly, it may be interpreted as an inverse filtering problem, so that the combination of the channel and equalizer must behave like ideal channel .The conventional equalizers such as Linear, Decision-feedback, and Fractionally- spaced equalizers are based on this approach. But their performance is poor especially when the channel response is non-linear. Secondly, the problem of equalization may be considered as a classification problem. All equalizers using neural networks are based on the latter approach..

The capabilities of neural networks for equalization of simple channels are described by Gibson, Siu and Cowan. Multi-layer perceptron (MLP) equalizers are superior to conventional linear and decision feedback equalizers in terms of probability of error, but their practical applications are severely restricted due to difficulties such as very long training time, indeterminate nature of training time, and lack of a methodology for architecture selection. Radial basis function network (RBFN) equalizer has received a great deal of attention because of its structural simplicity and more efficient learning compared to MLP. Moreover, there exists structural equivalence between RBFN equalizer and optimal symbol spaced Bayesian equalizer. However, the structure of RBFN equalizer depends on channel length, and thus requires a large number of centers in the hidden layer, which increases the computational complexity.

In this paper, we propose a new channel equalizer based on adaptive neuro-fuzzy inference system, i.e., an adaptive fuzzy inference system whose parameters are adapted using neural network learning algorithms. Herein hybrid learning algorithm is used for optimizing parameters of ANFIS. The performance of the proposed equalizer is evaluated in terms of bit-error rate (BER) for different noise powers in the channel. Both linear as well as non-linear channels are considered for performance evaluation. The BER of proposed equalizer is compared with conventional least mean square (LMS) equalizer, MLP equalizer, RBFN equalizer, and optimal Bayesian equalizer. Simulation results show that proposed equalizer has better BER performance compared to MLP and LMS equalizers, but slightly poorer than RBFN and optimal Bayesian equalizers. However, the proposed equalizer has smaller structure than RBFN equalizer, thus provides a better compromise between the performance and structural complexity. 


\section{Existing System}

High-speed link rates are entering the region where the bandwidth of the wires in cables or backplanes becomes severely limited by dielectric loss, skin-effect and impedance discontinuities. Additionally, in many applications the wires within a system can have significantly different channel characteristics. In these systems, achieving optimal performance for each link requires a flexible equalization solution that can adapt to the specific requirements of its channel.

In practice, the channel possibly distorts the transmitted signal before it reaches the receiver. It is then the receivers job to figure out what signal was transmitted, and to turn it into understandable information. In ideal scenario, the information the receiver delivers should coincide with the information fed into the transmitter. In a pass band signal, the process of distortion due to channel can be represented considering the frequency response of both signal as well as the channel. We consider that the signal has an ideal band limited spectrum, as shown in Fig. 1.

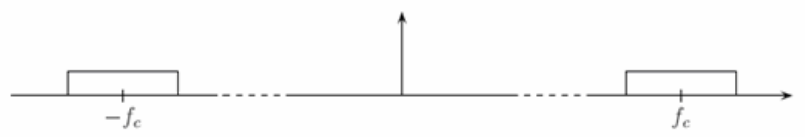

Fig. 1: Spectrum of Transmitted Pass band Signal But the channel may have a completely on-uniform spectrum, as can be represented by Fig. 2 .

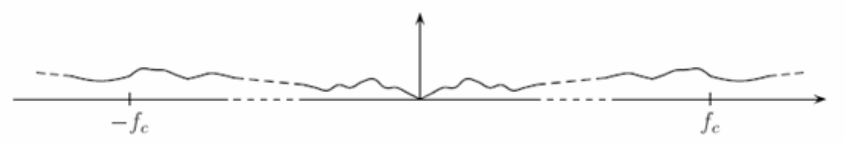

Fig. 2: Spectrum of the Channel Thus, the signal that is received at the receiver has a spectrum distorted by the channel.

This received signal spectrum can be represented as in Fig. 3

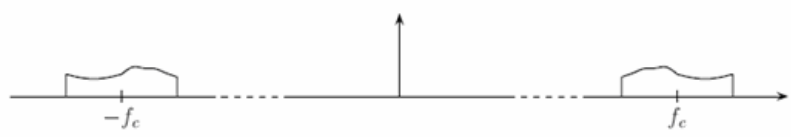

Due to this kind of distortion by the channel, the transmitted pulses will be smeared out so that the pulses corresponding to different symbols will not be separable. This phenomenon is known as intersymbol interference or ISI. The channel is said to be dispersive. To restore the transmitted sequence, some sort of signal processing must be performed at the receiver. This necessitates the use of channel equalization for proper data reception.

\subsection{Problem Definition}

In Communication channels due to various non-idealities like Inter Symbol Interference (ISI), Additive White Gaussian Noise (AWGN), Non-linear effects etc the input signal suffers from distortions which include Amplitude Distortion, Phase / Frequency Distortion and Non-linear Distortion. In order to neutralize the effect of these distortions introduced by the channel and to be able to recover the original signal as best as possible, we need Channel Equalizer which acts as a Compensator for the Channel Distortion.

Normally there are three methods used for Channel Equalization:

- Maximum Likelihood Sequence Detection

- Linear Equalization

- Non-Linear Equalization

But these methods can not give optimum performance in case of a channel having unknown or time varying characteristics like time varying delay affected by Inter Symbol Interference (ISI) and Additive White Gaussian Noise (AWGN). To solve this problem, we need an equalizer which is also time-varying and can adapt itself to the changes in channel characteristics.

\subsection{Problem Description}

By regarding the entire channel from the symbol sequence to the sampled measurements as a discrete-time system, it is possible to model the channel as:

$y(\mathrm{t})=\mathrm{h}_{\mathrm{o}}^{\mathrm{t}} \mathrm{d}(\mathrm{t})+\sum_{k=1}^{m} \mathrm{~h}_{k}^{\mathrm{t}} \mathrm{d}(\mathrm{t}-\mathrm{k})+\mathrm{n}(\mathrm{t})$ for all $\mathrm{t}>0$

Here $\left\{h_{k}^{t}\right\}_{k=0}^{m}$ is the time varying impulse response of the equivalent discrete time channel and $\boldsymbol{n}(\boldsymbol{t})$ is the additive noise introduced in the process of transmission-reception. 
Now, in order to recover the input $\boldsymbol{d}(\boldsymbol{t})$ from the received signal $\boldsymbol{y}(\boldsymbol{t})$, it can be clearly seen from the model represented in (1) that the signal $\boldsymbol{d}(\boldsymbol{t})$ is distorted by noise $\boldsymbol{n}(\boldsymbol{t})$ seen from the model represented in (1) that the signal $\boldsymbol{d}(\boldsymbol{t})$ is distorted by noise. $\sum_{k=1}^{m} h_{k}^{t} \mathrm{~d}(\mathrm{t}-\mathrm{k})$. To extract the original signal from this $\boldsymbol{y}(\boldsymbol{t})$, we must use an equalizer. The equalizer characteristics obviously depend upon the impulse response of the channel. Since this is not only unknown but also time varying, we must use an adaptive equalizer.

\subsection{Equalization problem}

The performance of a communication system is limited by ISI and additive Gaussian noise. Additive Gaussian noise is introduced in the signal traveling through channel and due to the thermal characteristics of the transmitter and receiver circuitry. Its effect can be reduced by operating at the high signal-to-noise ratio through the channel ${ }^{[}$So, the performance of high speed digital communication system operating over large Signal to Noise Ratio (SNR) is mainly limited by ISI. ISI is usually a result of the restricted bandwidth allocated to the channel and/or the presence of multipath distortion in the medium through which the information is transmitted. It causes the overlapping of the adjacent symbols of the transmitted signal and thus tends to limit the maximum data rate achievable through that channel. Such channels may be characterized by a finite impulse response (FIR) digital filter and an additive noise source

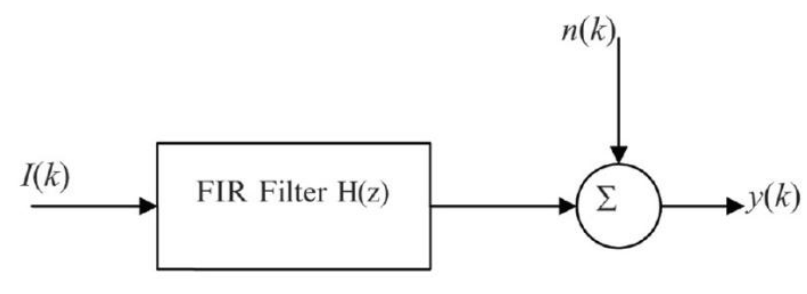

Figure3: Discrete-time Channel Model

The observed sequence $\{y(k)\}$, at the receiver in response to the input sequence $\{I(k)\}$ is

$$
\begin{aligned}
y(K) & =\sum_{\mathrm{n}=0}^{\mathrm{N}_{\mathrm{b}}-1} \mathrm{~h}(\mathrm{n}) \mathrm{I}(\mathrm{k}-\mathrm{n})+{ }^{\eta}(\mathrm{K}) \\
& =\mathrm{h}(0) \mathrm{I}(\mathrm{k})+\sum_{\mathrm{n}=0}^{\mathrm{N}_{\mathrm{b}}-1} \mathrm{~h}(\mathrm{n}) \mathrm{I}(\mathrm{k}-\mathrm{n})+{ }^{\eta}(\mathrm{K})
\end{aligned}
$$

where $\mathrm{N}_{\mathrm{h}}$ is the length of the channel impulse response and $\eta(k)$ is additive white Gaussian noise (AWGN) with zero mean. In the above equation, first term represents the scaled version of desired symbol, and second and third terms represent the effects of ISI and noise, respectively To reduce the effects of ISI and noise, the observed sequence $\{y(k)\}$ is applied to an equalizer of order $m$, whose structure is shown in Figure4

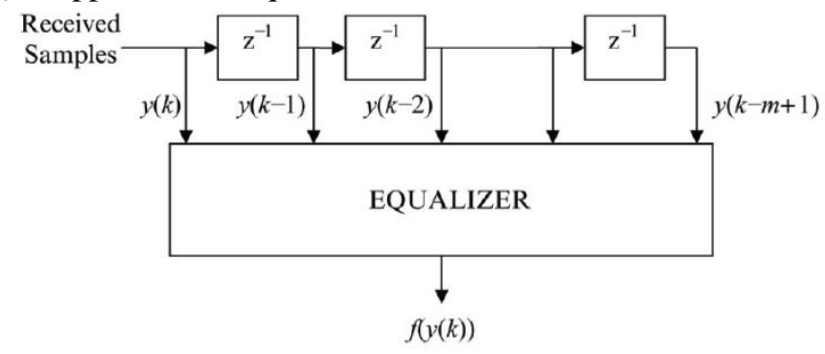

Figure4: Equalizer of order $m$

It consists of a tapped delay line with tap spacing equal to the symbol interval T. So, at each time instant $k$, the input to the equalizer is a vector containing $m$ most recent samples of the received signal $y(k)$. The equalizer performs some complex computations over this input vector in order to reduce ISI and noise

\subsection{Problem Formulation}

One of effective way for development of adaptive equalizers for nonlinear channels is the use of fuzzy technology in their development. For adaptive equalization of channel distortion the fuzzy equalizer is proposed. Human experts determine the fuzzy rules using input-output data pairs of the channel. These rules are used to construct the filter for nonlinear channel. The RLS and LMS algorithms are applied to change parameters of the membership functions of rules and develop equalizers. Using linguistic and numerical information the fuzzy adaptive filters are developed. The use of such approach improves the adaptation speed. 
In some case the construction of fuzzy rules for equalizers is difficult. In this case one of the effective technologies for construction of equalizer's knowledge base is the use of neural network. In this paper the channel equalization by using neuro-fuzzy network is considered. The use of neuro-fuzzy technology allows using small number of parameters, fast and easy train equalizer. The equalizer based on neural networks doesn't need appropriate knowledge about channel dynamics. These equalizers give better results in bit error rate (BNR), at the cost of computational strength.

\section{Proposed System}

We proposed that the application of neuro-fuzzy system for equalization of channel distortion is considered. The structure and operation algorithm of neuro-fuzzy equalizer are described. The use of neurofuzzy equalizer in digital signal transmission allows decreasing training time of parameters and decreasing the complexity of the network. The simulation of neuro-fuzzy equalizer is performed. The obtained result satisfies the efficiency of application of neurofuzzy technology in channel equalization.

\subsection{Introduction}

\section{Equalisation Using Neural Networks}

Numerous advances have been made in developing intelligent systems, inspired by biological neural networks. Researchers from many scientific disciplines are designing artificial neural networks (ANNs) to solve a variety of problems in pattern recognition, Function approximation, prediction, optimization, associative memory, and control.

Conventional approaches have been proposed for solving these problems. Although successful applications can be found in certain well-constrained environments, none is flexible enough to perform well outside its domain. ANNs provide exciting alternatives, and many applications could benefit from using them.

\subsubsection{Definition of Neural network}

A neural network is a machine that is designed to model the way in which the brain performs a particular task or function of interest. To achieve good performance, they employ a massive interconnection of simple computing cells referred to as 'Neurons' or 'processing units'.

A neural network is a massively parallel distributed processor made up of simple processing units, which has a natural propensity for storing experimental knowledge and making it available for use. It resembles the brain in two respects:

1. Knowledge is acquired by the network from its environment through a learning process.

2. Interneuron connection strengths, known as synaptic weights, are used to store the acquired knowledge.

\subsubsection{Importance}

It is apparent that a neural network derives its computing power through, first, its massively parallel distributed structure and, second, its ability to learn and therefore generalize. The use of neural networks offers the following useful properties and capabilities:

- Massive parallelism

- Distributed representation and computation

- Learning ability

- Generalization ability

- Input-output mapping

- Adaptively

- Uniformity of Analysis and Design

- Fault tolerance

The advent of neural networks marked the modeling of nonlinear adaptive systems which could provide high degree of precision, fault tolerance and adaptability compared to other forms of mathematical modeling. So the artificial neural networks are predominantly used for equalization. The Back Propagation (BP) algorithm is the best known and widely used learning algorithm for training ANNs since its proposal by Rumelhart and LeCun.

\subsubsection{Need for nonlinear equalizers}

The main reason nonlinear equalizers are preferred over their linear counterpart is that the linear equalizers do not perform well on channels which have deep spectral nulls in the pass band. In an attempt to compensate for the distortion, the linear equalizer places too much gain in the vicinity of the spectral nulls, thereby enhancing the noise present in these frequencies. 
Non-linear equalizers outperform the linear equalizers in terms of BER. Also the linear equalizers view equalization as inverse problem while non-linear equalizers view equalization as a pattern classification problem.

Consider the following example of the channel states for the two channels.

$$
\begin{aligned}
& \mathrm{H}_{1}(\mathrm{Z})=1+\mathrm{O} .5 \mathrm{Z}^{-1} \\
& \mathrm{H}_{2}(\mathrm{Z})=0.3842+0.8704 \mathrm{Z}^{-1}+0.3842 \mathrm{Z}^{-2}
\end{aligned}
$$

\subsection{NEURAL NETWORK EQUALISER}

These neural networks construct a functional relationship between input and output patterns through the learning process, and memorize that relationship in the form of weights for later application. The neural network equalizer outperforms the Linear Transversal filters in terms of bit error rate (BER) as most of the communication channels requires nonlinear decision. The structure of neural network equalizer is shown in the figure5.

In the figure $\mathrm{r}(\mathrm{k})$ represents received signal. The structure constitutes three significant parts- one input layer, a set of hidden layers, one output layer. All the nodes are interconnected by the weights $\mathrm{w}_{\mathrm{ij}}{ }^{1}$. Where $\mathrm{i}$ represents the destination node and $\mathrm{j}$ represents the source node. The superscript gives 1 the layer number.

An equalizer of order $m$ implies that it has $m$ input nodes in its input layer as shown in the figure5. An equalizer will have a single node in its output layer. The signal received sequentially is allowed to propagate through the hidden layers up to the node in the output layer.

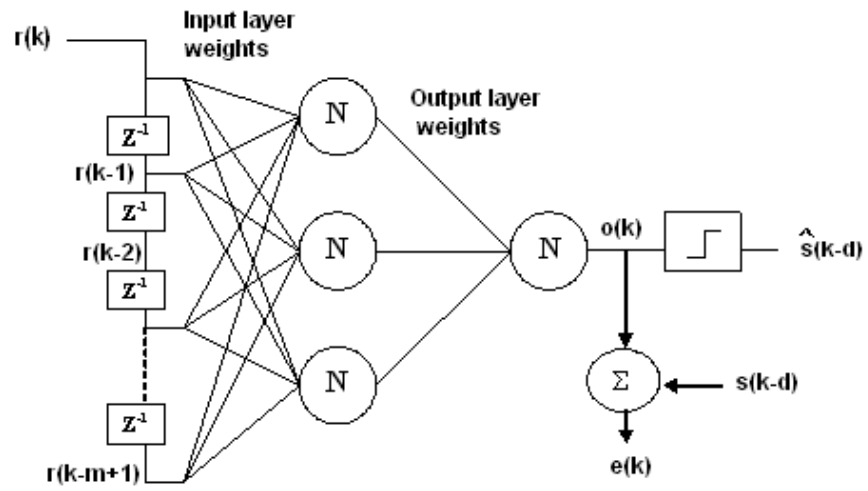

Figure5: Neural Network Equalizer

The output of each node is the weighted sum of outputs of all the nodes in the previous layer and affected by the activation function, which here is the hyperbolic tangent function given by

$$
\varphi(x)=\frac{1-\mathrm{e}^{-a x}}{1+\mathrm{e}^{-a x}}
$$

Where 'a'represents the slope of the activation function.

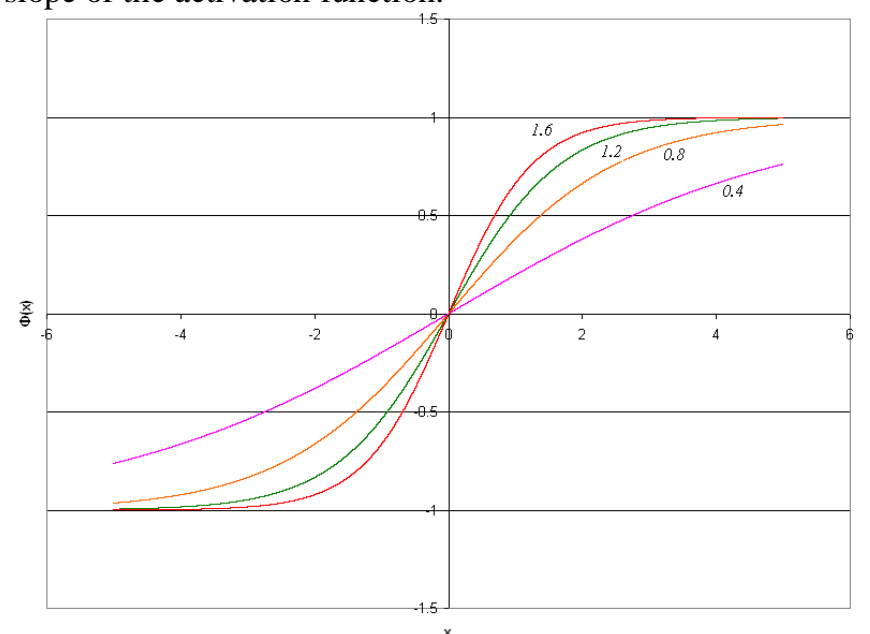

Figure6:Hyperbolic Tangent Function with varying slopes 
The figure 6 shows the hyperbolic tangent activation function with slope varying at $0.4,0.8,1.2$ and 1.6. As slope increases the function tends towards threshold function. The function attains a maximum value of +1 at and a minimum value of -1 at . Hence the output of each node of the neural network will be in the range $(-1,1)$. Since we are using binary PAM signal which takes either of the two values -1 or +1 , this function is very much suitable for equalization process. Mathematically the forward propagation of the neural network is given by

$$
\begin{aligned}
& v_{i}^{l}=\sum_{j=1}^{N_{l-1}}\left(w_{i j}^{l-1} \cdot y_{j}^{l-1}\right) \\
& y_{i}^{l}=\varphi\left(v_{i}^{l}\right)
\end{aligned}
$$

Where $\mathrm{v}_{\mathrm{i}}^{1}$ is called the induced local field or activation potential of node $\mathrm{i}$ in layer 1 and $\mathrm{N}_{\mathrm{l}-1}$ is the number of neurons in the layer (1-1) .

\subsection{Back Propagation Algorithm}

The BP algorithm is a very good example for the Gradient-based algorithms. The BP algorithm consists of two passes through the different layers of the network: a forward pass and a backward pass. In the forward pass, as mentioned earlier, an activity pattern (input vector) is applied to the sensory nodes of the network and its effect propagates through the network layer by layer. Finally an output is obtained at the output node as the actual response of the network. During the forward pass the synaptic weights are kept constant. In the backward pass, on the other hand, the synaptic weights are all adjusted in accordance with the error correction rule. The error signal, which is obtained by comparing the output of the node in the output layer with the desired response, is allowed to pass against the direction of synaptic weights (hence the name back propagation algorithm for this training process) and local gradients at each node is computed as given by

$$
\partial_{j}^{l}=\varphi^{\prime}\left(v_{j}^{l}\right) \cdot \sum_{i=1}^{N_{i+1}}\left(\partial_{i}^{l+1} \cdot w_{j i}^{l}\right)
$$

The local gradient depends on whether neuron $\mathrm{j}$ is an input node or a hidden node.

\subsection{DECISION FEEDBACK EQUALISATION}

The advantage of using the decision feedback equalizer is that ISI is eliminated without enhancement of noise by using past decisions to subtract out a portion of the ISI in addition to the feed forward filter ; a disadvantage is that decision error tend to propagate because they result in residual ISI and a reduced noise margin against noise at future decisions.

\subsubsection{Need for decision feedback}

There are some channels for which the negative centers and positive centers are very near and overlap in many cases due to the presence of additive noise. Such channels are called overlapping channels. One of the good examples of such a channel is $\mathrm{H}_{3}(\mathrm{z})=0.4084+0.8164 \mathrm{z}^{-1}+0.4084 \mathrm{z}^{-2}$

The channel state diagram for this channel $\mathrm{d}=1$ for is shown in the Figure 7

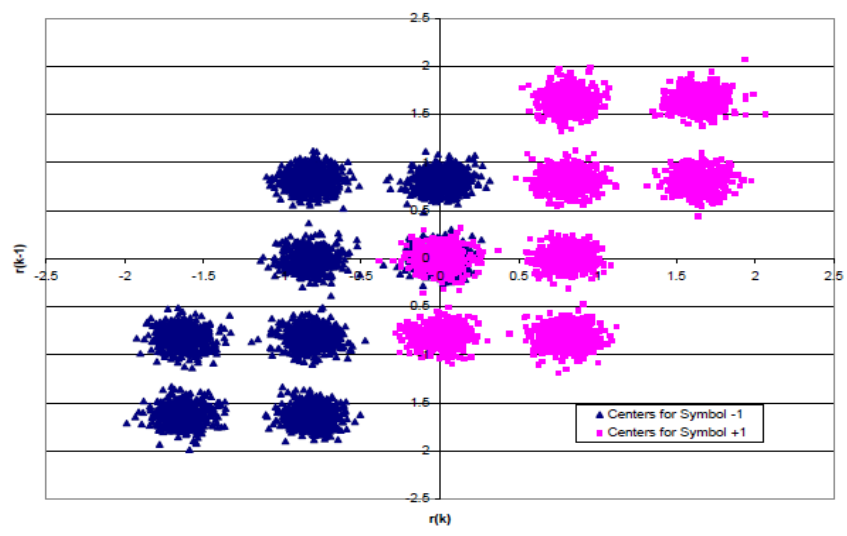

Figure 7:Channel state diagram for overlapping channel $\quad H 3(z)$.

Hence we need a decision feedback equalizer to classify these overlapping patterns. Earlier it is stated that equalization process can be considered as a classification problem and the advantage of using the feedback concept is that it reduces the number of states for decision making and hence ease the classification. 


\subsubsection{Decision Feedback Neural Network Equalizer}

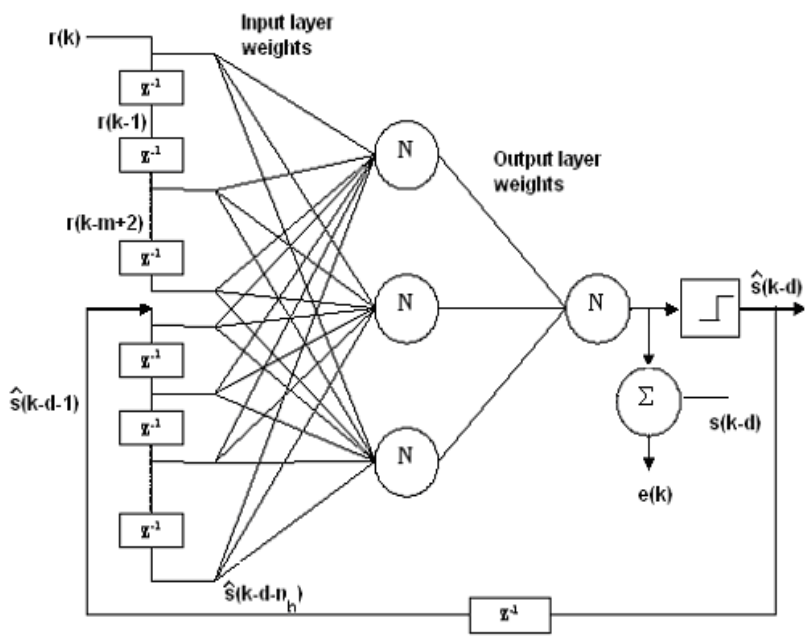

Figure8: Decision Feedback Neural Network Equalizer

The structure of the decision feedback neural network equalizer is shown in Fig8. It can be noted from figure that it is very much similar to the simple neural network equalizer except the inclusion of additional taps for feedback elements. This feedback is called decision feedback as we are feeding back the previous decisions. Number of feedbacks included is called feedback order $\mathrm{n}_{\mathrm{b}}$. Then the number of additional delay element required is $n_{b}-1$.

Hence, now, the total number of nodes in the input layer becomes .in the absence of feedback number the transmitted symbols that influence the equaliser decision are

$\left\{\mathrm{S}(\mathrm{k}), \mathrm{S}(\mathrm{k}-1) \ldots, \mathrm{S}\left(\mathrm{k}-\mathrm{m}-\mathrm{n}_{\mathrm{a}}+2\right)\right\}$

Thus the channel input sequence has $n_{s}=2^{m+n}{ }_{a}^{-1}$ combinations among which $n_{s} / 2$ constitute centers belonging to symbol +1 and the remaining $n_{s} / 2$ are for centers corresponding to symbol -1 . Hence here all the $n_{s}$ states are required for decision making. If we include feedback then the number of transmitted symbols that influence the equalizer performance are

$\left\{\left(\mathrm{S}(\mathrm{k}), \mathrm{S}(\mathrm{k}-1), . . \mathrm{S}\left(\mathrm{k}-\mathrm{m}-\mathrm{n}_{\mathrm{a}}+2\right)\right),\left(\hat{S}(\mathrm{k}-\mathrm{d}-1) . . \hat{S}\left(\mathrm{k}-\mathrm{d}-\mathrm{n}_{\mathrm{a}}\right)\right)\right\}$

Hence the feedback vector has $n_{f}=2^{n}{ }_{b}$ states. As a result of feedback, only a fractional number of these states, $n_{s} / n_{f}$ are needed for decision making. It can be noted that it is sufficient to employ a feedback order $\mathrm{n}_{\mathrm{b}}$, $n_{b}=n_{a}+\mathrm{m}-2-\mathrm{d}$

the performance of the neural network equalizer without feedback is very poor. But if we use feedback for this channel, the BER performance will improve.

\section{The Anfis Equaliser}

ANFIS is a fuzzy inference system whose parameters are optimized using the given input/output data set by using hybrid learning algorithm. The network-type structure of an ANFIS is similar to a neural network. It maps inputs through input membership functions and associated parameters, and then through output membership functions and associated parameters to outputs. In this paper, grid partitioning of training data is used to identify the initial structure of the ANFIS.

\subsection{ANFIS Architecture}

Illustrates an architecture of a two input single output ANFIS. Fixed and adaptive nodes are represented by circles and squares respectively.

Fuzzy if-then rules describing operation of the ANFIS may be written as

Rule 1: Rule 2 :

If $\mathrm{x}$ is $\mathrm{A}_{1}$ and $\mathrm{y}$ is $\mathrm{B}_{1}$, then $\mathrm{z}_{1}=\mathrm{p}_{1} \mathrm{x}+\mathrm{q}_{1} \mathrm{y}+\mathrm{r}_{1}$

If $x$ is $A_{2}$ and $y$ is $B_{2}$, then $z_{2}=p_{2} x+q_{2} y+r_{2}$. 


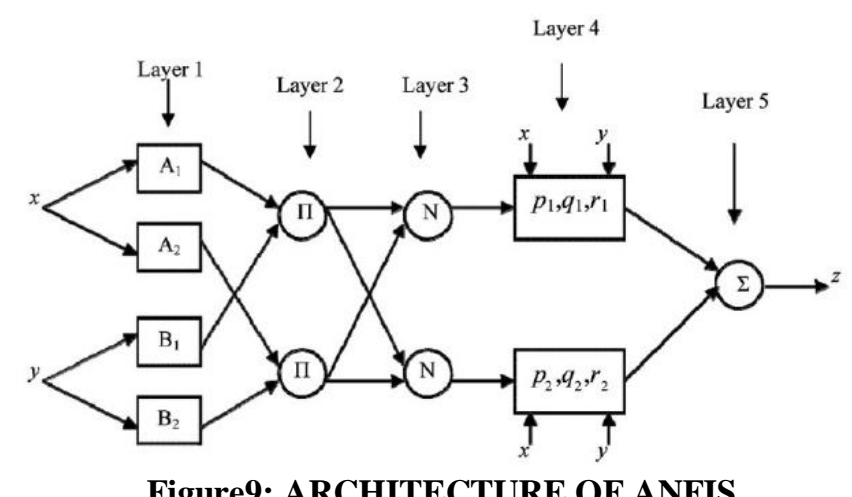

Figure9: ARCHITECTURE OF ANFIS

where $A_{i}$ and $B_{i}$ are the fuzzy sets in the antecedent, and $p_{i}, q_{i}$ and $r_{i}$ are the consequent parameters that are determined during the training process.

The ANFIS consists of the following five layers:

Layer1: Every node $\mathrm{i}$ in the first layer has a node function $\mathrm{Q}_{1, \mathrm{i}}$ which represents the output of the ith node in layer1.

$$
\begin{array}{r}
\mathrm{Q}_{1, \mathrm{I}}=\mu_{A_{i}}(\mathrm{x}) \text { for } \mathrm{i}=1,2 \\
\mu_{B_{i-2}}(\mathrm{y}) \text { for } \mathrm{i}=3,4
\end{array}
$$

For nodes 1 and 2, $\mathrm{x}, \mathrm{A}_{\mathrm{i}}$ and $\mu_{\mathrm{Ai}}$ are the input, linguistic term, and the associated membership function, respectively, whereas $\mathrm{y}, \mathrm{B}_{\mathrm{i}-2}$, and $\mu_{\mathrm{Bi}-2}$ are the input, linguistic term and the associated membership function for nodes 3 and 4 , respectively.

Layer 2: Each node output represents the firing strength of a rule and performs the fuzzy AND operation. The output of a node $i$ in this layer is the product of all the incoming signals for the $i$ th rule and is given by $Q_{2, i}=w_{i}=\mu_{A_{i}}(\mathrm{x}) \mu_{B_{i}}(\mathrm{y})$, for $\mathrm{i}=1,2 \quad------(14)$

Layer 3: An $i^{\text {th }}$ node in this layer calculates the ratio of the $i^{\text {th }}$ rule's firing strength to the sum of all rules' firing strengths. The output of node $i$ in this layer is the normalized firing strength, denoted by $w_{i}$, and given below $Q_{3, i}=\overline{w_{i}}=\frac{w_{i}}{w_{1+w_{2}}}, \mathrm{i}=1,2$

Layer 4: In this layer, every node has a node function given by $Q_{4, i}=\overline{w_{i}} z_{i}=\left(p_{i}+q_{i}+r_{i}\right)$, for $\mathrm{i}=1,2$

where $w_{i}$ is the normalized firing strength for node $i$ in layer 3 , and $\left\{p_{i}, q_{i}, r_{i}\right\}$ is the consequent parameter set of node i, corresponding to layer 4.

Layer 5: This is an output layer having single node with the output function $Q_{5}$, given by the summation of all the incoming signals

$\mathrm{Q}_{5}=\sum_{i} \bar{w}_{i} z_{i}=\frac{\sum_{i} w_{i} z_{i}}{\sum_{i} w_{i}}$

A block diagram of the ANFIS based channel equalizer.The input to the equalizer is the observed vector $\mathrm{y}(\mathrm{k})=\{\mathrm{y}(\mathrm{k}) \mathrm{y}(\mathrm{k}-1) \ldots \ldots \mathrm{y}(\mathrm{k}-\mathrm{m}+1)\}$ corrupted by ISI and AWGN corresponding to the transmitted signal I $(\mathrm{k})$ and $\hat{\mathrm{I}}(\mathrm{k})$ at the output of the equalizer is an estimate of the transmitted signal $\mathrm{I}(\mathrm{k})$.

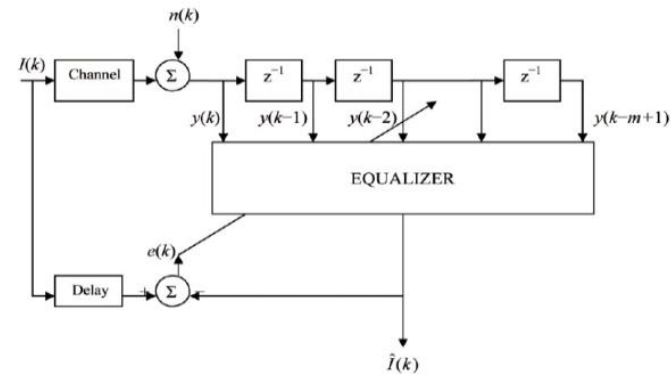

Figure10: The ANFIS Based Channel Equalizer 
The parameters of ANFIS equalizer are optimized using hybrid learning algorithm to minimize the error $e(k)$ between $I(k)$ and the corresponding estimate $\hat{I}(k)$. In the ANFIS architecture, if the values of the premise parameters associated with $A_{1}, A_{2} B_{1}$ and $B_{2}$ are fixed, then using Eqs, the overall output $Q_{5}$ may be expressed as:

$Q_{5}=\frac{w_{1}}{w_{1}+w_{2}} z_{1}+\frac{w_{2}}{w_{1}+w_{2}} z_{2}$

$=\overline{w_{1}}\left(p_{1} \mathrm{x}+q_{1} \mathrm{y}+r_{1}\right)+\overline{w_{2}}\left(p_{2} \mathrm{x}+q_{2} \mathrm{y}+r_{2}\right.$

$=\left(\bar{w}_{1} \mathrm{x}\right) p_{1}+\left(\bar{w}_{1} \mathrm{y}\right) q_{1}+\left(\bar{w}_{1}\right) r_{1}+\left(\bar{w}_{2} \mathrm{x}\right) p_{2}+\left(\bar{w}_{2} \mathrm{y}\right) q_{2}+\left(\bar{w}_{2}\right) r_{2}$

where $p_{1}, q_{1}, r_{1}, p_{2}, q_{2}$, and $r_{2}$ are the consequent parameters.

Similarly, when the values of consequent parameters are fixed, the use of Eqs yields the overall output $Q_{5}$ as given next

$$
\begin{aligned}
Q_{5} & =\frac{w_{1}}{w_{1}+w_{2}} z_{1}+\frac{w_{2}}{w_{1}+w_{2}} z_{2} \\
& =\frac{\mu_{A_{1}} \cdot \mu_{B_{1}} \cdot z_{1}+\mu_{A_{2}} \cdot \mu_{B_{2}} \cdot z_{2}}{\mu_{A_{1}} \cdot \mu_{B_{1}}+\mu_{A_{2}} \cdot \mu_{B_{2}}}
\end{aligned}
$$

where $\mu_{\mathrm{Ai}} \& \mu_{\mathrm{Bi}}$ are the membership functions corresponding to the inputs $\mathrm{x}$ and $\mathrm{y}$, respectively, assumed to be bell shaped in this paper and are given by the above equ, respectively.

$$
\mu_{A_{i}}=\frac{1}{1+\left\{\left(x-c_{A, i} \mid a_{A, i}\right)^{2}\right\} b_{A, i}}
$$

and

$$
\mu_{B_{i}}=\frac{1}{1+\left\{\left(y-c_{B, i} \mid a_{B, i}\right)^{2}\right\} b_{B, i}}
$$

where the premise parameters $\mathrm{a}_{\mathrm{A}, \mathrm{i}}, \mathrm{b}_{\mathrm{A}, \mathrm{i}}$ and $\mathrm{c}_{\mathrm{A}, \mathrm{i}}$ are associated with input $\mathrm{x}$, and $\mathrm{a}_{\mathrm{B}, \mathrm{i}}, \mathrm{b}_{\mathrm{B}, \mathrm{i}}$ and $\mathrm{c}_{\mathrm{B}, \mathrm{i}}$ with input $\mathrm{y}$. It may be easily observed that the overall output $\mathrm{Q}_{5}$ is (i) a linear function of the consequent parameters, if the premise parameters are fixed, and (ii) a non-linear function of the premise parameters if the consequent parameters are kept constant. Therefore, hybrid learning algorithm is appropriate for training the equalizer. This algorithm uses the least square algorithm for optimizing the consequent parameters and gradient decent algorithm for optimizing the premise parameters.

\subsection{Hybrid Learning Algorithm}

The training set used for optimization of the ANFIS equalizer parameters by using the hybrid learning algorithm consists of a set of source symbols $I(k)$ and the corresponding observed vectors $y(k)$. Let the overall parameters of the ANFIS are divided into two sets, $S_{1}$ and $S_{2}$, where

$S_{1}=$ set of premise (nonlinear) parameters,

$S_{2}=$ set of consequent (linear) parameters.

The Hybrid learning algorithm proceeds in an iterative manner through the following two passes: Forward Pass: In the forward pass of the hybrid learning algorithm, inputs are applied to layer 1 and propagated forward until the outputs of layer 4 are computed and then consequent parameters $S_{2}$ are identified by the least-square method.

Backward Pass: In this pass, the error signal propagates backward and the premise parameters $S_{1}$ are updated by the gradient descent method.

Table1:The Hybrid Learning Algorithm

\begin{tabular}{|l|l|l|}
\hline & Forward pass & Backward Pass \\
\hline $\begin{array}{l}\text { Premise } \\
\text { Parameters }\end{array}$ & Fixed & Gradient descent \\
\hline $\begin{array}{l}\text { Consquent } \\
\text { Parameters }\end{array}$ & $\begin{array}{l}\text { Least Square } \\
\text { Estimator }\end{array}$ & Fixed \\
\hline Signals & Node Outputs & Error Signal \\
\hline
\end{tabular}




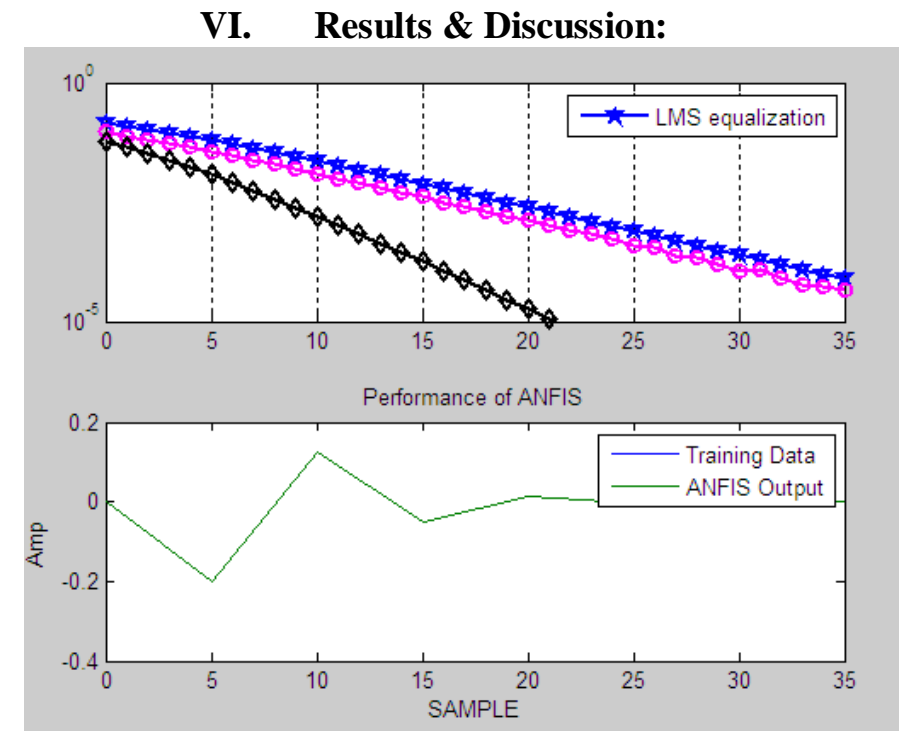

Simulation results show that the proposed equalizer outperforms MLP and LMS equalizers in terms of BER performance for both the linear and non-linear channels. The BER performance of the proposed equalizer remains almost same for both the linear and non-linear channels but that of MLP and LMS equalizers degrade further for the non-linear channel. Although the BER performance of the proposed equalizer is slightly poor than that of RBFN and optimal Bayesian equalizers, it has an advantage of having smaller structure with 40 hidden units and a total of 104 parameters only compared to 64 hidden units and a total of 384 parameters in both RBFN and Bayesian equalizers, and thus provides a better compromise between performance and structural complexity.

\section{Conclusion:}

In this paper a new equalizer based on ANFIS is proposed. Its performance is evaluated in terms of BER, and performance is compared with channel equalization using LMS, MLP, RBF, Bayesian classification technique.

\section{References:}

[1]. H Qureshi, "Adaptive equalization", Proceedings IEEE, Vol.73, pp. 1349-87, Sep 1985.

[2]. I S Chen, B Mulgrew, and P Grant, "A clustering technique for digital communication channel equalization using RBFN", IEEE Trans. Neural Networks, Vol. 4, pp. 570-9, Jul 11993.

[3]. J Lee, C D Beach, and N Tepedelenlioglu, "Channel equalization using radial basis function network", in IEEE International Conf. on Neural Networks, Washington D.C. USA, pp. 1924-8, Jun 1996.

[4]. B Mulgrew, "Applying radial basis functions", IEEE signal processing magazine, Vol. 13, pp.50-65, Mar 1996.

[5]. G J Gibson, S Siu, and C F N Cowan, "Adaptive equalization of finite non-linear channels using multi-layer perceptrons", IEEE Trans. Signal Processing, Vol. 20, No. 2, pp. 107-20, Jun 1990.

[6]. B Lu, and B L Evans, "Channel equalization by feedforward neural networks" in IEEE International Symposium on Circuits and Systems, Orlando, FL, USA, pp. 587-90, Jun 1999.

[7]. S Hyakin, "Communication sytems", $4^{\text {th }}$ ed, Singapur: John Wiley \& Sons (Asia) Pte Ltd.; 2001

[8]. R Abiyev, F Mamedov, and T Al-shanableh, "Analysis \& Design of Intellegent Systems using Soft Computing Techniques: Nonlinear neuro-fuzzy network for channel equalization, 2007", Berlin, Heidelberg: Springer-Verlag; 2007.

[9]. J S R Jang, C T Sun, and E Mizutani, "Neuro-fuzzy and soft computing: A Computational Approach to Learning and Machine intelligence", Englewood Cliffs: Prentice-Hall; 1997.

[10]. J G Proakis, "Digital communication", $4{ }^{\text {th }}$ ed, Singapore: McGraw Hill Publication; 1997.

[11]. R J Jhy-Shing, "ANFIS: Adaptive-Network-Based Fuzzy Inference System", IEEE Transaction on Systems, Man, and Cybernetics, Vol. 23, May 1993.

[12]. R Abiyev, and T Al-shanableh, "Neuro-Fuzzy Network for Adaptive Channel Equalization", Proceedings of the Fifth Mexican International Conference on Artificial Intelligence (MICAI 06), pp. 237-44, Nov 2006. 